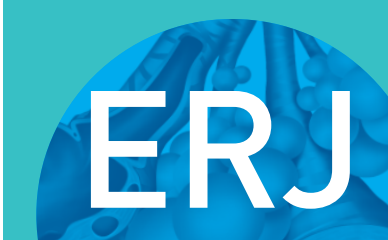

open research

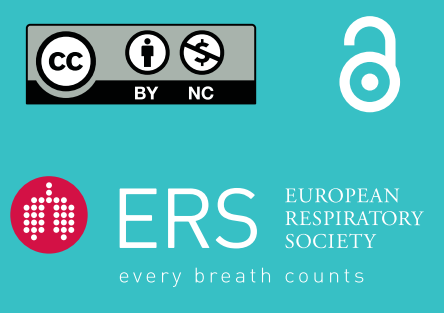

\section{Airway obstruction is associated with reduced variability in specific parts of the tidal breathing flow-volume curve in young children}

\section{To the Editor:}

Lung function testing in subjects with limited cooperation, such as young children, the elderly or the disabled, is often extremely challenging even when using methods that need a low level of cooperation like impulse oscillometry (IOS). Hence, measurement and analysis of tidal breathing have been studied as an alternative option for conventional lung function methods in detecting airway obstruction. However, attempts to determine clinically useful obstruction-related tidal breathing indices, such as peak points or line fits from the tidal breathing flow-volume (TBFV) curves, have produced very limited outcomes. A more recent approach has focused on looking at the tidal breathing curve variability over time. The tidal breathing indices and the mathematical methods for their quantification analysed in early studies have been diverse; for instance, approximate entropy $[1,2]$ and noise limit of the flow signal [3], interquartile range of various tidal breathing indices $[4,5]$, and coefficient of variability of tidal volume [6]. A common finding has been that lower airway obstruction is associated with reduced variability in the tidal breathing pattern.

Impedance pneumography is a method that enables continuous measurement of TBFV curves during sleep at night $[7,8]$. The aim of this study was to determine if the obstruction-related reduction in tidal breathing pattern variability is contained within a specific part of the TBFV curve by analysing nocturnal impedance pneumography measurements from wheezy (asthmatic) and healthy young children.

Two groups of subjects were recruited. The patients were selected based on three physician-witnessed lower airway obstruction episodes and were initially prescribed a 3-month period of inhaled corticosteroid (ICS) to gain control according to the Finnish guideline. Treatment was then stopped and 4 weeks after withdrawing the ICS, one nocturnal impedance pneumography recording was performed at home. The control group included only healthy children with a very low risk of asthma based on family and medical history, clinical examination, and lung function (IOS). The control group performed one to three measurements at home on consecutive nights. Patients visited Tampere University Hospital (Tampere, Finland) between September 2014 and March 2017, and controls visited Children's Hospital Srebrnjak (Zagreb, Croatia) between April 2017 and June 2017. The study was approved by the local ethics committees and parents/legal guardians provided signed, written, informed consent prior to any procedure. Due to the scarcity of previous data, the sample size was not based on formal power calculation.

The impedance signals were recorded using commercial equipment (Ventica Recorder; Revenio Research Ltd, Vantaa, Finland) and university prototype devices [9]. The devices were compared in a laboratory bench test to confirm interchangeable signals. All recordings were processed using the same commercially available software (Ventica Analytics 2.0.1; Revenio Research Ltd) and the same custom analysis scripts for analysis steps 3-6 below (MATLAB R2017A; MathWorks Inc., Natick, MA, USA).

@ERSpublications

Impedance pneumography recordings during sleep at night show reduced variability in specific parts of the expiratory flow-volume curve in children with recurrent wheeze http://bit.ly/ 2Wkfap0

Cite this article as: Seppä V-P, Hult A, Gracia-Tabuenca J, et al. Airway obstruction is associated with reduced variability in specific parts of the tidal breathing flow-volume curve in young children. ERJ Open Res 2019; 5: 00028-2019 [https://doi.org/10.1183/23120541.00028-2019]. 
The variability in the TBFV curves of each overnight recording was quantified by the following steps: 1) automatically discarding sections with a distorted impedance pneumography signal, 2) averaging TBFV curves in 5-min moving windows, 3) extracting specific volume range of the curves (e.g. 0-30\% inspiratory volume), 4) calculating linear correlations for each possible pair of averaged TBFV curves (using only the extracted range), 5) calculating the interquartile range (IQR) of the correlations, and 6) repeating from step 3 with another curve range $(5-35 \%, 10-40 \%$, etc.). Thus, a high correlation IQR value is associated with high variability in the specific TBFV curve range and low IQR with low variability during the recording night. The IQR values for each range of the curve were compared between the healthy controls and the patients using the Wilcoxon rank-sum test.

79 measurements were obtained from 39 healthy controls (age median (range) 4.3 (1.5-6.0) years) and 61 measurements from 61 patients (age $2.5(0.9-5.7)$ years). The variability of the TBFV curves was found to be higher during the inspiratory than the expiratory part of curves (figure 1). The variability was not significantly different between the controls and the patients in the first half of inspiration or in the very early part of the expiration, but for other parts, it was significantly reduced in the patients. The reduction in variability was clearly most significant at $15-45 \%$ of exhaled volume with a p-value of $10^{-8}$. Discriminative power was not associated with the level of variability.

Our results corroborate previous studies [1-6] by showing that airway obstruction is associated with reduced variability of the tidal breathing pattern. However, this is the first study to investigate which specific parts of the TBFV curves are affected, indicating the largest effect during the latter part of the first half of the expiration.

During normal tidal breathing, the activation of the inspiratory muscles does not stop abruptly at the end of inspiration. Instead, their activation decays slowly during expiration while opposing the elastic recoil of the lungs and the chest wall. SHEE et al. [10] found that in healthy adults, a reduction of $50 \%$ of post-inspiratory muscle activity takes on average $23 \%$, and ceases completely around $79 \%$ of the expiratory time. This implies that the TBFV shape in the early expiration is affected by the neural respiratory control, whereas the latter part of the expiration becomes progressively more passive, and the TBFV shape is determined predominantly by the combination of respiratory impedance and the elastic recoil forces.

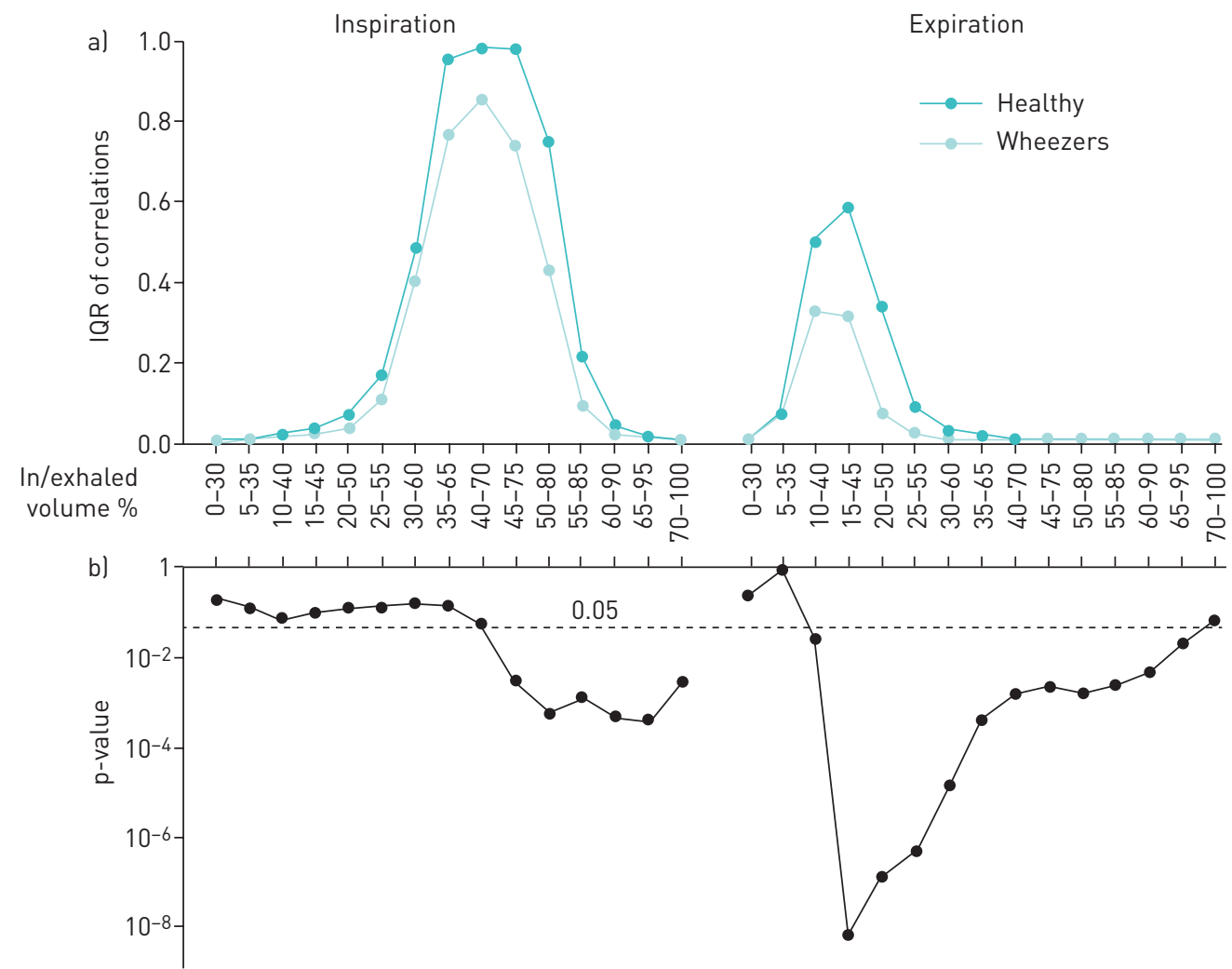

FIGURE 1 a) Median values of tidal breathing flow-volume (TBFV) curve shape variability (interquartile range (IQR) of correlations) in different parts of the TBFV curves for healthy children and patients. b) Significance ( $p$-value) of difference in variability between the two groups. The volume ranges refer to relative in/exhaled volume with $0 \%$ denoting the beginning of an inspiration or expiration. 
There are at least two potential mechanisms for a reduction in TBFV variability associated with obstruction. Firstly, obstruction results in a faster decay of the post-inspiratory muscle activity [11, 12], which changes the early expiration from controlled to uncontrolled and passive, and thus, presumably, renders it less variable. Secondly, the overall decrease of variability in respiration can be attributed to the reduction of degrees of freedom in the respiratory system under obstruction [13].

The study has some limitations. Sleep stages influence respiratory neural control [14] and respiratory mechanics [15] but due to lack of electroencephalographic assessment, we could not investigate their effect. In addition, there was a small age mismatch between the healthy control and patient groups, but the TBFV variability did not correlate with age in either group. Furthermore, we did not have a reference method to confirm the presence or lack of airway obstruction during the measurement nights.

We conclude that the obstruction-related reduction in the variability of the TBFV curve occurs in its specific parts, being the most discriminative at $15-45 \%$ of the expired volume in young children with recurrent wheeze.

Ville-Pekka Seppä $\odot^{1}$, Anton Hult ${ }^{1}$, Javier Gracia-Tabuenca ${ }^{2}$, Marita Paassilta ${ }^{3}$, Jari Viik $\oplus^{2}$, Davor Plavec $^{4,5}$ and Jussi Karjalainen ${ }^{3}$

${ }^{1}$ Revenio Research Ltd, Vantaa, Finland. ${ }^{2}$ Faculty of Medicine and Health Technology, Tampere University, Tampere, Finland. ${ }^{3}$ Allergy Centre, Tampere University Hospital, Tampere, Finland. ${ }^{4}$ Research Dept, Srebrnjak Children's Hospital, Zagreb, Croatia. ${ }^{5}$ Medical Faculty, University J.J. Strossmayer, Osijek, Croatia.

Correspondence: Ville-Pekka Seppä, Revenio Research Ltd., Äyritie 22, Vantaa, Finland. E-mail: vps@iki.fi

Received: Jan 292019 | Accepted after revision: April 272019

Conflict of interest: V-P. Seppä has three patents with royalties paid by Revenio Group Inc. and is an employee of Revenio Group Inc., which commercialises impedance pneumography technology. A. Hult is an employee of Revenio Group Inc., which commercialises impedance pneumography technology. J. Gracia-Tabuenca has nothing to disclose. M. Paassilta has nothing to disclose. J. Viik has three patents with royalties paid by Revenio Group Inc. D. Plavec reports a grant and consultancy fees from Revenio Group during the conduct of the study, and grants from GlaxoSmithKline, Boehringer Ingelheim, Novartis and Chiesi, grants and personal fees from MSD, and personal fees from AbbVie, Sandoz, GlaxoSmithKline, Salveo, Menarini/Berlin Chemie and Salvus, outside the submitted work. J. Karjalainen reports personal fees from AstraZeneca, Boehringer Ingelheim, Chiesi, GlaxoSmithKline, Mundipharma, Novartis, Orion Pharma and Teva, outside the submitted work.

Support statement: This work was supported by Tampereen Teknillinen Yliopisto and Tampereen Tuberkuloosisäätiö. Funding information for this article has been deposited with the Crossref Funder Registry.

\section{References}

1 Dames KK, Lopes AJ, de Melo PL. Airflow pattern complexity during resting breathing in patients with COPD: Effect of airway obstruction. Respir Physiol Neurobiol 2014; 192: 39-47.

2 Veiga J, Lopes AJ, Jansen JM, et al. Airflow pattern complexity and airway obstruction in asthma. J Appl Physiol 2011; 111: 412-419.

3 Seppä V-P, Pelkonen AS, Kotaniemi-Syrjänen A, et al. Tidal flow variability measured by impedance pneumography relates to childhood asthma risk. Eur Respir J 2016; 47: 1687-1696.

4 Hmeidi H, Motamedi-Fakhr S, Chadwick E, et al. Tidal breathing parameters measured using structured light plethysmography in healthy children and those with asthma before and after bronchodilator. Physiol Rep 2017; 5: 13168.

5 Hmeidi H, Motamedi-Fakhr S, Chadwick EK, et al. Tidal breathing parameters measured by structured light plethysmography in children aged 2-12 years recovering from acute asthma/wheeze compared with healthy children. Physiol Rep 2018; 6: e13752.

6 Usemann J, Suter A, Zannin E, et al. Variability of tidal breathing parameters in preterm infants and associations with respiratory morbidity during infancy: a cohort study. J Pediatr 2019; 205: 61-69.

7 Seppä V-P, Pelkonen AS, Kotaniemi-Syrjänen A, et al. Tidal breathing flow measurement in awake young children by using impedance pneumography. J Appl Physiol 2013; 115: 1725-1731.

8 Malmberg LP, Seppä V-P, Kotaniemi-Syrjänen A, et al. Measurement of tidal breathing flows in infants using impedance pneumography. Eur Respir J 2017; 49: 1600926.

9 Vuorela T, Seppä V-P, Vanhala J, et al. Design and implementation of a portable long-term physiological signal recorder. IEEE Trans Inf Technol Biomed 2010; 14: 718-725.

10 Shee CD, Ploy-Song-Sang Y, Milic-Emili J. Decay of inspiratory muscle pressure during expiration in conscious humans. J Appl Physiol 1985; 58: 1859-1865.

11 Citterio G, Agostoni E, Santo AD, et al. Decay of inspiratory muscle activity in chronic airway obstruction. J Appl Physiol 1981; 51: 1388-1397.

12 Morris MJ, Madgwick RG, Frew AJ, et al. Breathing muscle activity during expiration in patients with chronic airflow obstruction. Eur Respir J 1990; 3: 901-909.

13 Raoufy MR, Ghafari T, Darooei R, et al. Classification of asthma based on nonlinear analysis of breathing pattern. PLOS ONE 2016; 11: e0147976. 
14 Horner RL. Respiratory physiology: central neural control of respiratory neurons and motoneurons during sleep. In: Kryger M, Roth T, Dement W, eds. Principles and Practice of Sleep Medicine. 5th Edn. Philadephia, Saunders, 2010; pp. 237-249.

15 Carroll JL, Donnelly DF. Respiratory physiology and pathophysiology during sleep. In: Kryger M, Roth T, Dement W, eds. Principles and Practice of Sleep Medicine. 5th Edn. Philadephia, Elsevier Saunders, 2010; pp. 179-192. 